\title{
Serotonin syndrome in burns patients
}

\author{
A Kenny, W Kleintjes \\ Corresponding author: Wayne Kleintjes (waynekleintjes@yahoo.com)
}

Summary: This case report illustrates the pitfalls of abnormal neurocognitive behaviour in a burns patient and reviews the vagaries of the diagnosis and treatment of serotonin syndrome.

S Afr J Surg 2018;56(3)

http://dx.doi.org/10.17159/2078-5151/2018/v56n3a2456

A 16-year-old boy who sustained $45 \%$ total body surface area (20\% partial thickness, $15 \%$ full thickness, $10 \%$ inhalation) flame burns was admitted to our Burn Centre on 14 June 2016, via Victoria Hospital where he received emergency treatment including escharotomy on the right lower limb. His injury was sustained on 11 June 2016 during a house fire in which other individuals died. His injuries were mostly to the lower part of his body but included partial thickness burns to arms, face and head.

His initial treatment included the standard protocols of the unit viz:-complete clinical examination - including appropriate special investigations, Ventilator Associated Pneumonia (VAP) care, head up, ulcer prophylaxis, sedation, analgesia, appropriate venous and arterial access, etc.

The patient was septic with raised inflammatory markers, positive blood cultures, foul-smelling wounds and bilateral infiltrates on chest $\mathrm{x}$-ray; he was initiated on appropriate antibiotics. He underwent multiple surgical debridements and skin grafts for source control as well as definitive treatment during his stay in the unit.

During August he exhibited signs and symptoms in keeping with major depressive disorder and was seen by a psychologist in response to an interdepartmental referral. A decision was made to commence $30 \mathrm{mg}$ daily oral Fluoxetine (Selective Serotonin Reuptake Inhibitor - SSRI) treatment.

Mid-August, during a dressing change, the intern doctor noted clonus of the lower limbs and investigated thoroughly. His chemical pathology was within normal limits. He exhibited no other signs or symptoms of serotonin syndrome viz:- altered mentation, diarrhoea, nausea and or vomiting. The autonomic disregulation he experienced could be attributed to pain, sepsis and the hypermetabolic response to burn pathology, but the clonus not. Therefore, clonus in the severely burned patient on multiple drug regimens (including analgesia, antiemetic, ulcer prophylaxis) on SSRI therapy, in the absence of other hard clinical signs of serotonin syndrome, should alert the clinician to the possibility of mild serotonin syndrome.

\section{Discussion}

A potentially life threatening condition known as serotonin syndrome poses a potential risk to patients admitted to a burns unit, the diagnosis of which can potentially be masked by symptoms commonly seen in burns patients.

Serotonin or 5-hydroxytriptamine is a monoamine neurotransmitter that is primarily found in the gastrointestinal tract, platelets and central nervous system of the human body. In the central nervous system, it has its main effect on mood, appetite, affective behaviour, food intake, thermoregulation, migraine, emesis, sexual behaviour and sleep, but also affects some cognitive functions like memory and learning. ${ }^{1,2,3,4,5}$

Serotonin is produced in the brainstem`s raphe nuclei and stored in presynaptic vesicles. ${ }^{1}$ Neuronal activation causes release of serotonin into the synapse. Reuptake of excess serotonin to the presynaptic vesicles is facilitated by an active transport mechanism, or it is metabolised by monoamine oxidase. The liver is responsible for systemic metabolism of serotonin.

A vast majority of pharmacological agents alter serotonin levels (Table 1), of which the most common would be selective serotonin reuptake inhibitors (SSRIs) or monoamine oxidase inhibitors (MAOIs) both of which are used primarily for the treatment of depression. These drugs interfere with the serotonin pathway of the brain either by increasing serotonin synthesis, decreasing serotonin metabolism, increasing serotonin release, inhibition of serotonin reuptake or direct stimulation of the serotonin receptor; increased levels of serotonin is the end/therapeutic result., ${ }^{4,6}$

Serotonin syndrome is a predictable complication of excess serotonergic stimulation and is thus not an idiosyncratic reaction. $^{7}$

Overdosing with a single causative agent seldom causes a serotonin syndrome; however, more commonly coadministration of pro-serotonergic agents can lead to an augmented effect on serotonin levels. ${ }^{1,2,3,9}$

Serotonin syndrome is a cluster of signs and symptoms that develop usually within $6-8$ hours of initiation or alteration of a potentially causative agent, either therapeutic drug use, intentional overdosing or complex interactions between drugs that directly or indirectly modulate the serotonin system. ${ }^{1,4}$ Some studies have also shown an association between 
Table 1: Pharmacological agents that commonly cause Serotonin syndrome

\begin{tabular}{|c|c|}
\hline Drug indications & Pharmacological agent \\
\hline \multirow[t]{11}{*}{ Antidepressants } & Tricyclic antidepressants \\
\hline & SSRIs \\
\hline & SNRIs \\
\hline & Trazodone \\
\hline & Litium \\
\hline & Buspirone \\
\hline & Clomipramine \\
\hline & Venlafaxine \\
\hline & Phenelzine \\
\hline & Moclobemide \\
\hline & Monoamine oxidase inhibitors \\
\hline \multirow[t]{11}{*}{ Analgesia } & Pethidine \\
\hline & Tramadol \\
\hline & Fentanyl \\
\hline & Tapentadol \\
\hline & Morphine \\
\hline & Meperidine \\
\hline & Dextromethorphan \\
\hline & Methadone \\
\hline & Codeine \\
\hline & Oxycodone \\
\hline & Buprenorphine \\
\hline Anti-Migraine & Triptans (sumatriptan) \\
\hline \multirow[t]{4}{*}{ Stimulants } & Methylenedioxymethamphetamine (Ecstacy) \\
\hline & Lysergic acid diethylamide (LSD) \\
\hline & Cocaine \\
\hline & Methamphetamine \\
\hline \multirow[t]{3}{*}{ Antipsychotics } & Risperidone \\
\hline & Olanzapine \\
\hline & Mirtazapine \\
\hline \multirow[t]{2}{*}{ Anti-convulsants } & Valproate \\
\hline & Carbamazapine \\
\hline \multirow[t]{3}{*}{ Anti-emetics } & Granisteron \\
\hline & Metoclopromide \\
\hline & Odansetron \\
\hline Antiretrovirals & Ritonavir \\
\hline Antihistamines & Chlorpheniramine \\
\hline \multirow{4}{*}{$\begin{array}{l}\text { Herbal } \\
\text { medication }\end{array}$} & St John`s wort (hypericum perforatum) \\
\hline & $\begin{array}{l}\text { Syrian Rue (harmine and harmaline } \\
\text { (MAOI's)) }\end{array}$ \\
\hline & Panax ginseng (ginseng) \\
\hline & Nutmeg \\
\hline Anxiety disorders & Buspirone \\
\hline \multirow[t]{3}{*}{ Antibiotics } & Linezolide \\
\hline & Fluconazole (+ citalopram) \\
\hline & Augmentin (+ SSRI) \\
\hline
\end{tabular}

withdrawal of certain medications and the development of serotonin syndrome. ${ }^{1,10}$ The initial symptoms can be subtle and easily missed or ascribed to other causes, which is why the true incidence of serotonin syndrome is not known, but is thought to mirror the increasing number of serotonergic agents being used. ${ }^{1,11}$ Also influencing the questionable incidence numbers is the fact that $85 \%$ of general physicians are unaware of this syndrome as a diagnosis. ${ }^{1}$ The Toxic Exposure Surveillance System reported that $27.49 \%$ of patients who were exposed to SSRIs developed significant symptoms of serotonin syndrome of which $0.3 \%$ resulted in death. The overall mortality rate is reported to be $10-15 \%$ (Hall and Henry) ${ }^{8,12}$ Serotonin syndrome is reported to occur in $16 \%$ of patients who overdose on SSRIs. ${ }^{3}$

Symptoms can be subdivided into autonomic, neuromuscular and mental state, and the seriousness grouped as mild, moderate and severe/life threatening. ${ }^{4}$ (Figure 1)

The differential diagnosis for serotonin syndrome includes adrenergic crisis, the anticholinergic toxidrome and neuroleptic malignant syndrome. ${ }^{5,6,9}$ To differentiate between these syndromes, a thorough history, investigation of all medication and illicit drug use as well as a thorough physical examination is needed. The physical examination should especially focus on the neuromuscular system. ${ }^{1}$ Hyperkinesis is said to be the most distinctive and useful finding to diagnose serotonin syndrome..$^{5,6,9,10}$ A unique clinical sign that should guide you to the diagnosis of serotonin syndrome is lower limb hyperreflexia and clonus with normal upper limb reflexes. ${ }^{9}$

The diagnosis is primarily clinical and there is no single test to make the diagnosis. ${ }^{1,10,11}$ Clinical diagnosis is also complicated by the complexity of the symptoms and that not every patient will present with all the above symptoms. ${ }^{1}$ In an attempt to make diagnosing serotonin syndrome easier and more accurate, people have developed numerous criteria, some more useful than others and some more accurate than others. Currently the most agreed on criteria is that of the Hunter Area Toxicology Service (HATS) with a sensitivity of $84 \%$ and specificity of $97 \%$ (Figure 1 ) ${ }^{3,5}$

Treatment includes prompt recognition of the symptoms, discontinuation of the causative agents and supportive care, which includes fluid resuscitation, benzodiazepines for the treatment of myoclonus and hyperreflexia and external cooling for hyperthermia. In severe cases, sedation, neuromuscular paralysis and intubation should be considered. . $^{1,4,11,13}$ It is advised to avoid succinylcholine for paralysis if possible due to the risk of arrhythmias from hyperkalemia. ${ }^{1}$ There is no role for antipyretics in the management of serotonin syndrome as the hyperthermia is caused by the increased muscle activity and not by central causes.

The antihistamine Cyproheptadine, a serotonin inhibitor, should be considered in moderate cases and is recommended in severe cases, despite the lack of good evidence..$^{1,4,9}$ With effective treatment, the signs and symptoms of serotonin syndrome should resolve within 24-48 hours. ${ }^{6}$ Physical restraints are not advised as this may increase the risk of rhabdomyolysis, increased lactic acidosis and hyperthermia. ${ }^{1}$ If there is a need for physical restraints it should soon be 
Serotonin Syndrome Symptom Classification

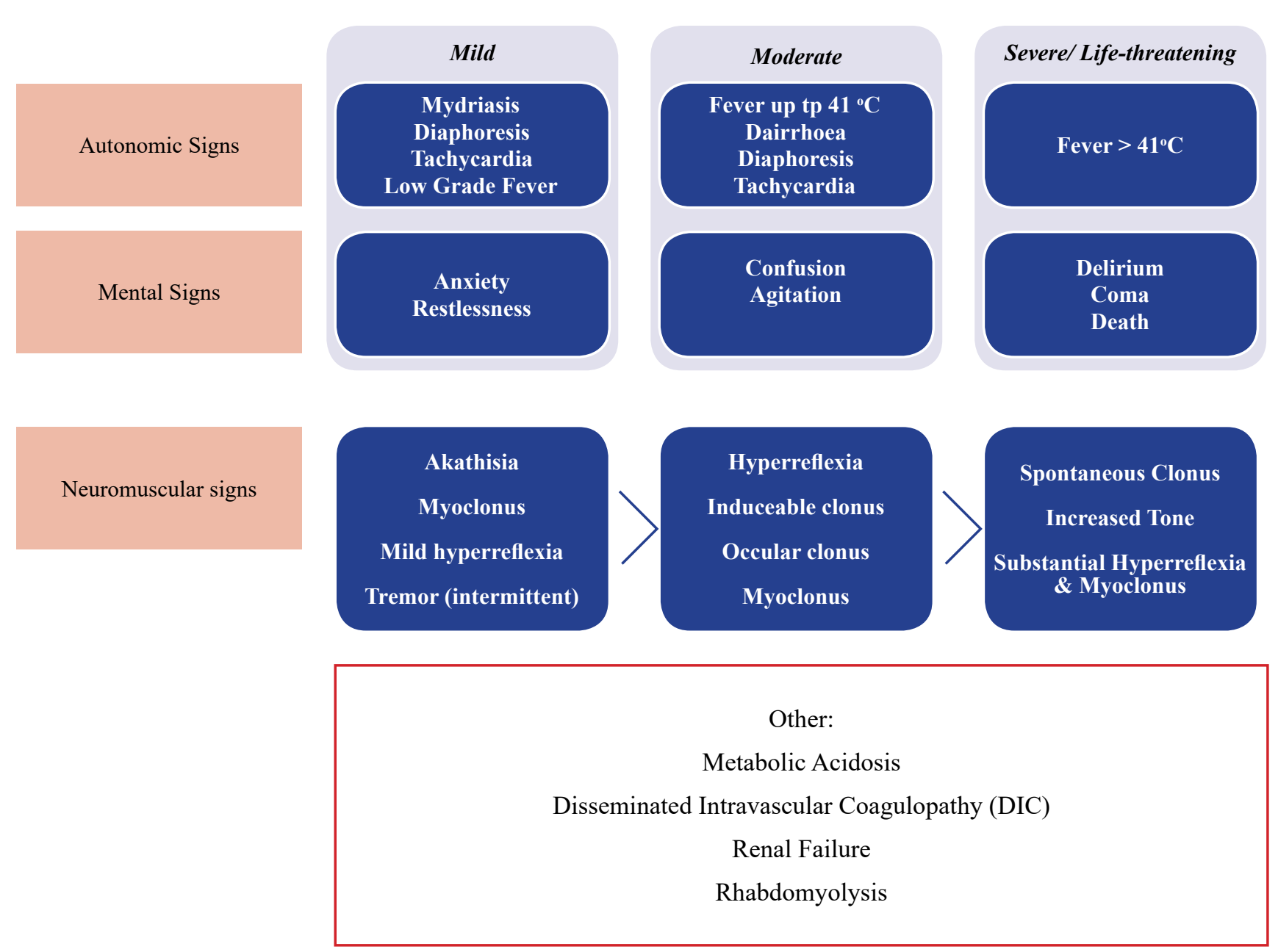

Figure 1. Serotonin Syndrome Symptom Classification

Compiled by information from Franc $C^{6}$ in combination with other resources ${ }^{1,3,4,8,9,11}$

\begin{tabular}{lll}
\multicolumn{3}{l}{ Table 2: Comparing symptoms of Serotonin syndrome and symptoms commonly seen in Burns patients } \\
\hline Symptoms & Serotonin syndrome & Possible symptoms seen in burns patients for different reasons \\
\hline Fever & + & + \\
Tachycardia & + & + \\
Diaphoresis & + & + \\
Diarrhoea & + & + \\
Tremors & + & + - \\
Hyperreflexia & + & + - \\
Clonus & + & + \\
Restlessness & + & + \\
Anxiety & + & + \\
Agitation & + & + \\
Delirium & + & + \\
Insomnia & + & \\
Data compiled from multiple publications $1,2,3,4,5$ &
\end{tabular}




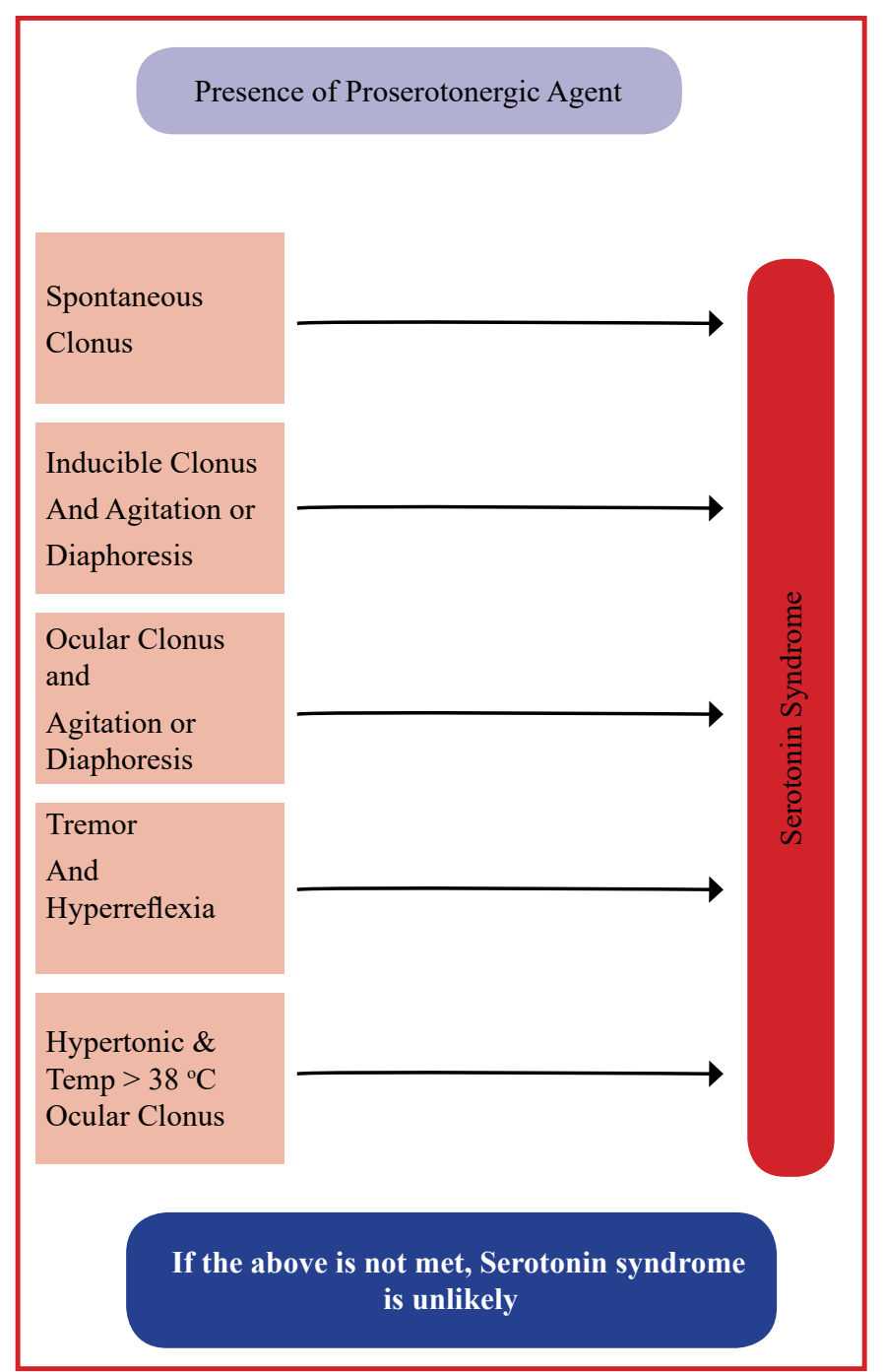

Figure 2. Diagnosis of Serotonin Syndrome. Compiled by information from Dunkley EJC3 and other resources ${ }^{5,8}$

replaced with chemical restraints as needed. ${ }^{1}$

Prevention of serotonin syndrome could be improved by continually educating physicians, altering prescribing practices and good pharmacological research. ${ }^{1,3,4}$

A high risk patient population for serotonin syndrome is those patients who are admitted to a burns unit. Pain management in burns patients are of utmost importance, and play a cardinal role in the management of the patient. Analgesia protocols differ between units, but the one thing that all units agree on is that a multimodal approach is needed to control pain adequately. This usually involves multiple drugs from different classes and different receptor activity.

Due to the nature of the injury, burns patients are at a higher risk of developing other conditions, like depression, anxiety, chronic pain syndromes, electrolyte disturbances and wound infection, all of which are amenable and deserving of appropriate treatment, which usually involves more pharmacological agents.

The risk of severe drug interactions is increased where more pharmacological agents are used, this being the case in almost every patient who is admitted with burn wounds.

The problem does, however, come in when the patient develops a serotonin syndrome, as the symptoms are easily masked by sedation and common symptoms seen in burns patients (Table 2).

Differentiating between serotonin syndrome and common symptoms in burns patients can be difficult for various reasons. The most important principle is to keep it in the back of your mind and exclude other causes for the symptoms. Careful prescription of pharmacological agents and a thorough drug history is of utmost importance and can guide your diagnosis and early treatment which can be lifesaving.

\section{REFERENCES}

1. Boyer EW, Shannon M. The serotonin syndrome. N Engl J Med. 2005;352(11):1112-20. Erratum in: N Engl J Med. 2005;356(23):2437.

2. Schenk M, Wirz S. Serotoninsyndrom und medikamentöse Schmerztherapie. Schmerz. 2015;29:229-251.

3. Dunkley EJC, Isbister GK. The hunter serotonin toxicity criteria: simple and accurate diagnostic decision rules for serotonin toxicity. Q J Med. 2003;96:635-642.

4. Dvir Y, Smallwood P. Serotonin syndrome: a complex but easily avoidable condition. Gen Hospital Psychiatry 30 (2008)284287.

5. Ganetsky M, Kavita M. Serotonin syndrome in dextromethorphan ingestion responsive to propofol therapy. Pediatric Emergency care. 2007;23:829-831.

6. Taylor JJ, Wilson JW. Linesolid and serotonergic drug interactions: A Retrospective survey. CID. 2006;43:180-186.

7. Frank C. Recognition and treatment of serotonin syndrome. Can fam physician. 2008;54(7):988-992.

8. Wolvetang T, Janse R. Serotonin syndrome after methylene blue administration during cardiac surgery: A case report and review. J Cardio and Vasc Anest. 2016;30(4):1042-45.

9. Thorpe EL, Pizon AF. Bupropion Induced Serotonin Syndrome: A case Report. J Med Toxicol. 2010;6:168-171.

10. Stevenson E, Schembri F. Serotonin syndrome associated with clozapine withdrawal. JAMA Neurol. 2013;70(8): 1054-55.

11. DeSilva KE, Le Fore DB. Serotonin syndrome in HIV-infected individuals receiving antiretroviral therapy and fluoxetine. AIDS. 2011;15:1281-5.

12. Devlin R, Henry JA. Clinical review: Major consequences of illicit drug consumption. Crit Care. 2008;12(1):202.

13. Katus LE, Frucht SJ. Management of serotonin syndrome and Neuroleptic malignant Syndrome. Curr Treat Options Neurol. 2016;18(9):39. 